\title{
Oxidation-Reduction Potential as a Biomarker for Severity and Acute Outcome in Traumatic Brain Injury
}

\author{
Kimberly B. Bjugstad, ${ }^{1}$ Leonard T. Rael, ${ }^{1}$ Stewart Levy, ${ }^{2}$ Matthew Carrick, ${ }^{3}$ \\ Charles W. Mains, ${ }^{4,5,6}$ Denetta S. Slone, ${ }^{1,5}$ and David Bar-Or ${ }^{1,4,5,6}$ \\ ${ }^{1}$ Department of Trauma Research, Swedish Medical Center, Englewood, CO 80113, USA \\ ${ }^{2}$ InterMountain Neurosurgery, St. Anthony Hospital, Lakewood, CO 80228, USA \\ ${ }^{3}$ Medical Center of Plano, Plano, TX 75075, USA \\ ${ }^{4}$ Department of Trauma Research, St. Anthony Hospital, Lakewood, CO 80228, USA \\ ${ }^{5}$ Department of Biomedical Sciences, Rocky Vista University, Aurora, CO 80134, USA \\ ${ }^{6}$ Penrose-St. Francis Health Services, Colorado Springs, CO 80907, USA
}

Correspondence should be addressed to David Bar-Or; dbaror@ampiopharma.com

Received 4 May 2016; Revised 12 July 2016; Accepted 17 July 2016

Academic Editor: Rodrigo Franco

Copyright (C) 2016 Kimberly B. Bjugstad et al. This is an open access article distributed under the Creative Commons Attribution License, which permits unrestricted use, distribution, and reproduction in any medium, provided the original work is properly cited.

\begin{abstract}
There are few reliable markers for assessing traumatic brain injury (TBI). Elevated levels of oxidative stress have been observed in TBI patients. We hypothesized that oxidation-reduction potential (ORP) could be a potent biomarker in TBI. Two types of ORP were measured in patient plasma samples: the static state of oxidative stress (sORP) and capacity for induced oxidative stress (icORP). Differences in ORP values as a function of time after injury, severity, and hospital discharge were compared using ANOVAs with significance at $p \leq 0.05$. Logit regression analyses were used to predict acute outcome comparing ORP, Injury Severity Score (ISS), Abbreviated Injury Scale (AIS), and Glasgow Coma Scale (GCS). Antioxidant capacity (icORP) on day 4 was prognostic for acute outcomes $(p<0.05)$. An odds ratio of 4.08 was associated with poor acute outcome when $i$ CORP $>7.25 \mu \mathrm{C}$. IcORP was a better predictor than ISS, AIS, or GCS scores. sORP increased in those with the highest ISS values $(p<0.05)$. Based on these findings ORP is useful biomarker for severity and acute outcome in TBI patients. Changes in ORP values on day 4 after injury were the most prognostic, suggesting that patients' response to brain injury over time is a factor that determines outcome.
\end{abstract}

\section{Introduction}

Currently, assessing the extent of injury and prognosticating eventual outcome of patients with traumatic brain injury (TBI) are difficult tasks. The Glasgow Coma Scale (GCS) and the Abbreviated Injury Scale (AIS) correlate with injury severity and have some prognostic power; however they rely on the subjective interpretation of patient injury by health care professionals, cerebral function, and can be influenced by age and other factors [1-6]. For these reasons, the Centers for Disease Control and Prevention has suggested that they are not be used in isolation when assessing the extent of injury or forecasting eventual patient outcome $[6,7]$.

Biomarkers may provide an unbiased and independent evaluation of TBI severity and patient outcome. Glial fibrillary acidic protein (GFAP), S100 $\beta$, and soluble urokinase plasminogen activator receptor (suPAR) have been used to assess survival in TBI patients [8-10]. Tau and GFAP have also been used to estimate cerebral function using GCS score at time of discharge, and the Glasgow Outcome Scale (GOS) score at 6 or 12 months $[4,8,11]$. Ubiquitin C-terminal hydrolase (UCH-1), matrix metalloproteinase 9 (MMP9), and MMP2 have been associated with TBI severity $[12,13]$. Most of these biomarkers can be measured from plasma or serum samples, minimizing the difficulty of obtaining a cerebrospinal fluid (CSF) sample but the process is still not adapted to fast turnaround times in the critical setting of TBI.

A cascade of events that lead to brain tissue ischemia and oxidative stress are evident in TBI. The peripheral and local immune systems respond by releasing proinflammatory 
cytokines, neutrophils, and reactive oxygen species (ROS); the latter then increases oxidant activity [14-18]. Cytogenic edema and the release of free glutamate potentiate oxidant activity by overwhelming mitochondrial energy through glutamate-induced excitotoxicity and inducing a state of oxidative stress $[19,20]$. Thus, a biomarker that associates with oxidative changes in brain injury could assist in the assessment of TBI patients.

Changes in oxidation-reduction potential (ORP), as an indicator of oxidative stress, might be a suitable biomarker for TBI. ORP is the net balance in activity between oxidants and reductants, also known as the redox potential. When oxidant activity exceeds reductant activity, the biological sample is under a state of oxidative stress [21-25]. ORP has been used for over 50 years when determining if the oxidant activity is sufficiently high enough in treated water to kill bacteria and other microbes [26-28]. Early studies in our lab and by others found changes in ORP in blood plasma samples from trauma patients, suggesting the benefit of measuring ORP in the biomedical arena [29-32].

Traditional methods for measuring ORP use large reusable platinum silver-silver chloride electrodes contained within a glass probe which is then submerged into a large volume of sample and analyzed using a galvanometer. The major limitations of this technology have been electrode contamination over time and the large sample volume [33]. Because of these limitations, the application of ORP to biomedical assessment has been limited. A novel technology has removed these limitations by developing a single-use disposable sensor requiring only $30 \mu \mathrm{L}$ of sample. This new system is sensitive enough to differentiate between oxidative states before and after exercise, to identify septic patients, and to measure changes in antioxidant activity in stored breast milk [34-38]. Using this novel technology, the present study explores the use of ORP as proxy of injury progression, severity, and survival in TBI patients.

\section{Methods}

2.1. Participant Information, Ethics, and Consent. Participants were a subset from a retrospective cohort study of multiple trauma/TBI patients previously reported [2]. Trauma patients were admitted to one of two level I trauma centers in the Denver-metro area-Swedish Medical Center (Englewood, CO) or St. Anthony Hospital (Lakewood, CO), between January 1, 2008, and December 31, 2012. The study was approved by the HealthOne HCA and the St. Anthony Hospital institutional review boards. Consent for daily blood draws was given by the patient or their legally authorized representatives. Twenty self-proclaimed healthy individuals were recruited as age-matched controls.

2.2. Inclusion Criteria. Patients $(n=132)$ with TBI were identified through the trauma registry (TraumaBase ${ }^{\circledR}$ database, Clinical Data Management) with one or more of these diagnostic injury codes based on the International Classification of Diseases, Ninth Revision, Clinical Modification (ICD9-CM): concussion (850.0-850.99); cerebral or cerebellar contusion or laceration (851.0-851.99); subarachnoid hemorrhage (852.0-852.19); subdural hemorrhage (852.2-852.39); extradural hemorrhage (852.4-852.59); other, unspecified intracranial hemorrhage (853.0-853.19); and intracranial injury of unspecified nature (854.0-854.19).

2.3. Exclusion Criteria. To identify those in which TBI was the primary injury, patients were excluded if the head AIS was less than two and if another AIS region was scored higher than the head AIS. Patients with less than five plasma samples obtained during hospitalization were also excluded. After exclusions, the final sample size was 104 (Table 1).

2.4. Plasma ORP Measurements. Whole blood was collected by venipuncture using heparinized Vacutainers during routine morning blood sampling. Samples were processed to plasma by centrifugation for 10 minutes at $1,000 \times \mathrm{g}$. Plasma was aliquoted and stored frozen at $-80^{\circ} \mathrm{C}$ until processing. Plasma ORP was measured using the RedoxSYS ${ }^{\circledR}$ system as a measure of the electron transfer from reductants (antioxidants) to oxidants under a constant negligible current (static ORP, sORP) and then by increasing the oxidative current (capacity ORP, cORP). For those interested in additional details regarding the RedoxSYS system, please see Rael et al. [39]. The sORP provides a measure of the current balance between all known and unknown oxidants and reductants/antioxidants. As such, higher sORP (in millivolts, $\mathrm{mV}$ ) suggests a higher level of oxidative stress. The cORP measures the biological sample's ability to withstand an oxidative insult by applying an increasing oxidizing current. This current ultimately exhausts all antioxidants present in the sample. cORP is expressed in microcoulombs $(\mu \mathrm{C})$. Typically the higher the cORP, the more the capacity a sample has to mitigate an oxidative insult but, because raw cORP data were not normally distributed, the inverse of each cORP value was generated to normalize the data; thus a higher icORP suggests a lower capacity to handle induced oxidative stress. While sORP and cORP are related, one looks at the current state of oxidative stress (sORP), while the other assesses the potential for oxidative stress (cORP).

2.5. Study Outcomes. The primary outcome was to determine if ORP could distinguish between discharge status and acute patient outcomes. Acute outcome was dichotomized based on hospital discharge status into good acute outcome (survival: discharged to home, skilled, or acute care facilities) and poor acute outcome (nonsurvival: patients who died or were transferred to hospice) (Table 2). Secondary outcomes included changes in ORP values over time after injury, TBI diagnosis, and degree of severity (Injury Severity Score (ISS) and GCS).

2.6. Statistical Analysis. Tables 1 and 2 provide descriptive statistics for the variables and outcomes of interest in the present study. All data were analyzed and graphed using Statistica (Dell, Inc.) or MedCalc. Differences in sORP and icORP between the TBI cohort and aged matched controls were analyzed by Student's $t$-test. Significance level of 
Table 1: Participant demographics.

\begin{tabular}{|c|c|c|c|}
\hline Demographic & Mean (95\% CI) & Median (IQR) & $N$ \\
\hline Age (years) & $54.53(50.0-59.0)$ & $55.5(34-76)$ & 104 \\
\hline AIS-head score & $3.85(3.7-4.0)$ & $4(3-5)$ & 104 \\
\hline ISS & $18.77(17.4-20.2)$ & $17(14-25)$ & 104 \\
\hline GCS & $11.27(10.3-12.3)$ & $14(7-15)$ & 91 \\
\hline ICU LOS & $6.18(5.1-7.3)$ & $4.5(2-9)$ & 104 \\
\hline Hospital LOS & $12.51(10.6-14.4)$ & $10(6.5-15)$ & 104 \\
\hline $\mathrm{ED}$, pulse & $86.81(83.0-90.7)$ & $86(73-96)$ & 102 \\
\hline ED, temperature $\left({ }^{\circ} \mathrm{F}\right)$ & $97.60(97.1-98.0)$ & $97.8(96.8-98.4)$ & 45 \\
\hline ED, respiratory rate & $16.77(15.5-18.0)$ & $16.5(14-20)$ & 102 \\
\hline ED, systolic BP & $139.38(134.5-144.2)$ & $134(124-154)$ & 102 \\
\hline $\mathrm{ED}$, diastolic $\mathrm{BP}$ & $79.79(76.0-83.60)$ & $82.5(71-89)$ & 52 \\
\hline ED, probability of survival & $0.86(0.82-0.91)$ & $0.96(0.83-0.98)$ & 91 \\
\hline sORP at admission & $187.78(183.0-192.5)$ & $185.9(172.2-205.2)$ & 89 \\
\hline$i$ cORP at admission & $4.42(4.2-4.7)$ & $4.46(3.5-5.3)$ & 89 \\
\hline Gender (ratio females: males) & \multicolumn{2}{|c|}{$37: 67$} & 104 \\
\hline Number of complications (\%) & & & 104 \\
\hline None & \multicolumn{2}{|c|}{$64.1 \%$} & 67 \\
\hline One & \multicolumn{2}{|c|}{$21.94 \%$} & 23 \\
\hline Two-five & \multicolumn{2}{|c|}{$13.9 \%$} & 14 \\
\hline
\end{tabular}

ED: emergency department; LOS: length of stay.

TABLE 2: Group categories used in statistical analyses.

\begin{tabular}{|c|c|}
\hline Type of TBI injury & \\
\hline Concussion (any participant with a concussion) & 4 \\
\hline SAH w/SDH/EDH (subarachnoid hemorrhage with any other hemorrhage) & 37 \\
\hline SAH alone & 23 \\
\hline SDH/EDH (subdural hemorrhage and/or epidural hematoma) & 15 \\
\hline Other (skull fracture, other non-ICH injury, anything else) & 22 \\
\hline Total & 101 \\
\hline ISS severity category & \\
\hline Mild/moderate $($ ISS score $<15)$ & 33 \\
\hline Severe (ISS score 16-25) & 51 \\
\hline Profound (ISS score > 25) & 20 \\
\hline Total & 104 \\
\hline GCS category & \\
\hline Minor $(\geq 13)$ & 57 \\
\hline Moderate (12-9) & 8 \\
\hline Severe $(\leq 8)$ & 26 \\
\hline Total & 91 \\
\hline Hospital discharge status & \\
\hline Good acute outcome & \\
\hline Home (home or home health) & 31 \\
\hline Intermediate (skilled nursing or rehabilitation facilities) & 57 \\
\hline Acute (long-term acute care) & 10 \\
\hline Poor acute outcome & \\
\hline Death/hospice (min-max hospital LOS: 6-19 days) & 6 \\
\hline Total & 104 \\
\hline
\end{tabular}


TABLE 3: Comparative values of the ROC analyses for icORP on day 4, ISS, AIS-head, and GCS at predicting acute outcome.

\begin{tabular}{|c|c|c|c|c|}
\hline & icORP day 4 & ISS & AIS-head & GCS \\
\hline Cut-off for survival status & $\geq 7.25 \mu C$ & $>27$ & $>4$ & $\leq 8$ \\
\hline ROC-AUC & $0.87^{*}(0.78-0.94)$ & $0.78^{*}(0.68-0.85)$ & $0.73^{*}(0.63-0.81)$ & $0.54(0.43-0.65)$ \\
\hline $\begin{array}{l}\text { Sensitivity } \\
\text { (\% of poor acute outcomes) }\end{array}$ & $33.33(0.8-90.6)$ & $33.33(4.3-77.7)$ & $50.0(11.8-88.2)$ & $40.0(5.3-85.3)$ \\
\hline $\begin{array}{l}\text { Specificity } \\
\text { (\% of good acute outcomes) }\end{array}$ & $100(95.2-100)$ & $89.90(82.0-95.0)$ & $75.51(65.8-83.6)$ & $72.1(61.4-81.2)$ \\
\hline Positive predictive value & $100(2.5-100)$ & $16.7(2.1-48.4)$ & $11.1(2.4-29.2)$ & $7.7(0.9-25.1)$ \\
\hline Negative predictive value & $97.4(90.9-99.7)$ & $95.7(89.2-98.8)$ & $96.1(89.0-99.2)$ & $95.4(87.1-99.0)$ \\
\hline Accuracy & $96 \%$ & $87 \%$ & $74 \%$ & $70 \%$ \\
\hline$N$ & 78 & 104 & 104 & 91 \\
\hline
\end{tabular}

$95 \%$ confidence intervals. ${ }^{*}$ Significant AUC values, $p<0.05$.

$p<0.05$ was used in all analyses. Unless indicated otherwise, all graphs are presented as mean \pm SEM.

Differences between discharge statuses were analyzed using ANOVA with Fisher LSD post hoc analyses when appropriate. Receiver operating characteristic (ROC) analysis was used to examine acute outcomes in terms of hospital survival based on ORP. The combined prognostic power of ORP, ISS, and AIS-head score was evaluated using forward logistic regression.

To gauge injury progression based on ORP values, data was analyzed for the first 14 days after injury. Data was then grouped into blocks of time after injury to analyze ORP changes long-term: day of injury (day $0,<24$ hours estimated postinjury time), first 48 hours, days 3-7 (week 1 ), days 8-14 (week 2), days 15-21 (week 3), and $\geq 21$ days (week 4 and beyond). Both analyses were done using one-way ANOVAs.

Group comparisons were used to determine changes in ORP as a function of the type of TBI and the degree of severity defined by ISS and GCS (Table 2). Analyses of ORP measures taken at admission, on day 4 and day 7 , were analyzed using separate one-way ANOVAs. Pearson's $r$ moment correlation was used between ORP values and ISS, AIS-head, and GCS values.

\section{Results}

3.1. Patient Demographics and ED Measures. The antioxidant capacity measures (icORP) taken on day 7 were significantly correlated to the verbal $(r=0.31, p<0.05)$ and motor $(r=0.27, p<0.05)$ portions of the GCS taken at admission, with increasing scores associated with increasing icORP values, thus decreasing antioxidant capacities. icORP values on day 7 and day 4 and sORP values on day 7 were negatively correlated with body temperature on arrival $(r=$ $-0.43, r=-0.37$, and $r=-0.42, p<0.05$, resp.), indicating that a higher temperature was associated with a later elevation in antioxidant capacities but also increased oxidative stress. Higher sORP on day 7 was also correlated with higher diastolic blood pressure $(r=0.37, p<0.05)$. ORP measures taken at admission and other day 4 measures were not correlated with ED measures (Table 1). No ORP value was not significantly different based on sex $(p>0.05$ all days) or whether the patient was ventilated $(p>0.05$ all days)

3.2. Estimating Hospital Discharge Status and Acute Outcome. The four categories of hospital discharge status were not significantly different in their sORP measures at admission, day 4 , or on day 7. Similar icORP levels across discharge statuses were also present at admission; however on day 4 those with a poor acute outcome (those who died or were transferred to hospice) had significantly higher icORP values, and thus lower antioxidant capacity, than those with a better acute outcome (Figure 1). This divergence of groups tended to remain through day 7 although not as strong, as suggested by the trend in significance $(p=0.11)$. Because of the significant effects found on day $4, i$ CORP data were used in ROC and logit regression analyses to determine its strength at predicting a poor or good acute outcome and were compared to the predictive power of ISS, AIS-head, and GCS values.

From the ROC analyses, the area under the curves (AUCs) for day 4 icORP values were significant $(p<0.05$; Table 3). An icORP cut-off value of $7.25 \mu \mathrm{C}$ generated a specificity of $100 \%$, indicating that all surviving patients had a day $4 i$ CORP less than or equal to $7.25 \mu \mathrm{C}$. The negative predictive power (NPV) was $97.4 \%$ suggesting that in patients with a day 4 icORP value less than $7.25 \mu \mathrm{C}$ it would be highly likely that they have a good acute outcome. ISS and AIS-head scores also had significant AUCs but with lower specificity, negative predictive power, and accuracy (Table 3 ). GCS scores failed to reach significance in this data set. The logit regression results indicate that only day 4 icORP values significantly contributed to the overall model $\left(X^{2}=5.78\right.$, $p<0.05)$ and had an odds ratio of 4.08. AIS-head and ISS values failed to enter the model.

3.3. Controls versus TBI and ORP Changes after Injury. Plasma samples from healthy controls had significantly lower sORP and icORP values than those measured in TBI patients (sORP: $t(107)=9.66, p<0.0001$; icORP: $t(104)=9.04$, $p<0.0001$; Figure 2).

In the first 48 hours after injury, sORP values increased and remained stable through at least the first 10 days after injury (Figure 2). Grouping the days after injury into blocks of time suggests that sORP values remain stable through at least 


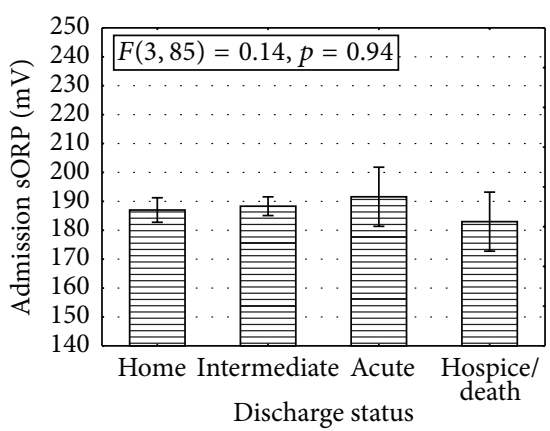

(a)

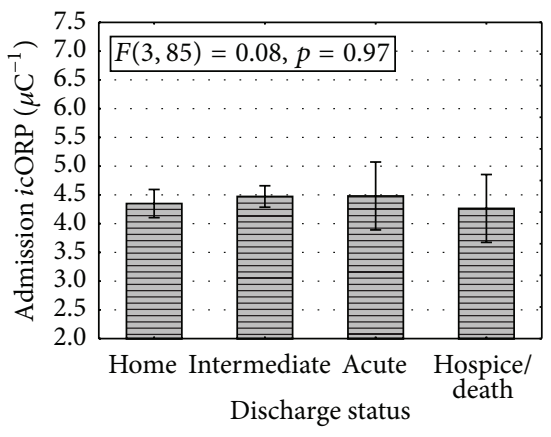

(d)

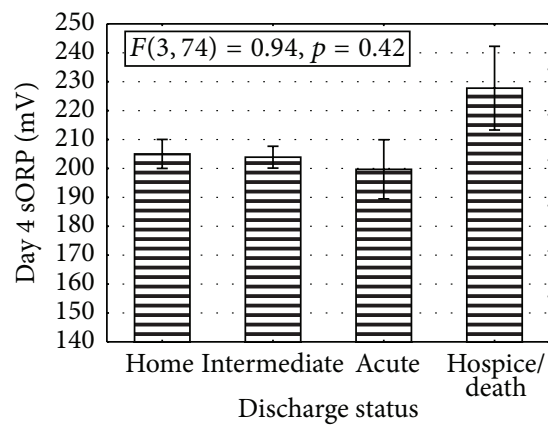

(b)

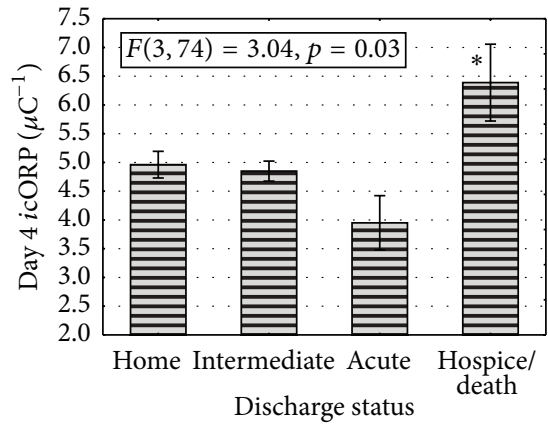

(e)

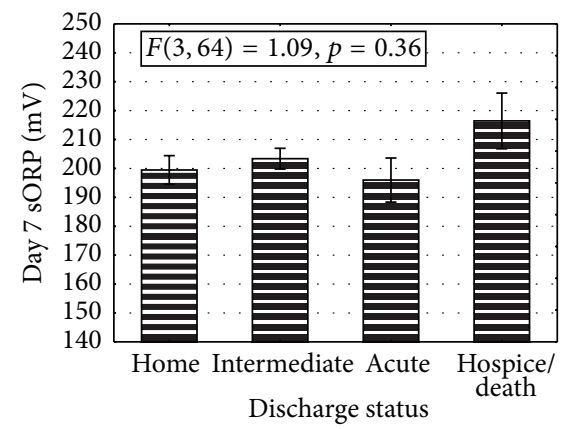

(c)

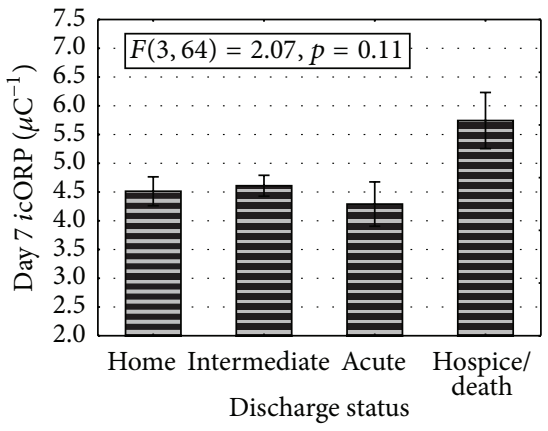

(f)

FIGURE 1: Assessment of survival based on hospital discharge can best be predicted by icORP on day 4. (a) Admission measures of $i$ cORP were not significantly different between patients with ultimately different discharge statuses. (b) By day 4 after injury, there was a significant difference between groups, with those that were ultimately discharged to a hospice or who died having much higher icORP values than any other discharge status. Those discharged to acute care facilities were the lowest but this failed to reach significance $(p=0.06$ and 0.08 between home and intermediate, resp.). (c) icORP values on day 7 were, again, not significantly different between the groups. (d) The ROC curve showing the AUC for day 4 icORP values. See Table 3 for ROC results. Data are presented as means \pm standard error of the mean (sem); * means significantly greater than all other groups.

TABLE 4: Correlations between sORP and icORP and traditional measures of severity.

\begin{tabular}{|c|c|c|c|c|c|c|}
\hline & \multicolumn{3}{|c|}{ sORPs } & \multicolumn{3}{|c|}{ icORP } \\
\hline & Admission & Day 4 & Day 7 & Admission & Day 4 & Day 7 \\
\hline ISS & 0.11 & $0.36^{*}$ & 0.15 & 0.05 & $0.28^{*}$ & -0.08 \\
\hline AIS-head & 0.10 & $0.36^{*}$ & 0.16 & 0.08 & $0.28^{*}$ & 0.00 \\
\hline GCS & -0.01 & -0.18 & 0.13 & -0.01 & -0.04 & $0.29^{*}$ \\
\hline
\end{tabular}

${ }^{*}$ Significant correlation; $p<0.05$.

21 days and then dropped significantly to levels below those measured in the first 48 hours (Figure 1(b)); however they were still significantly higher than controls levels $(t(76)=$ $7.02, p<0.05)$.

The icORP values revealed a similar pattern. TBI patients experienced an increase in icORP values (lower antioxidant capacity) in the first 24 hours and icORP values remained high through at least 6 days after injury (Figure 2). Antioxidant capacity began to return to more normal values after 21 days after injury; however the icORP values were still higher than those measured in healthy controls $(t(76)=4.79, p<$ $0.05)$.

3.4. Injury Severity. On day 4, there were significant correlations between ORP values and the ungrouped ISS, and AIShead scores (Table 4). Higher ISS and/or AIS-head scores were related to higher sORP and icORP values. GCS scores correlated only with day 7 icORP values, with increasing icORP associated with increasing GCS scores. Admission values failed to correlate.

In confirmation of the correlations, ORP measures on day 4 significantly distinguished between ISS groups (Figure 3). Patients with severe (ISS 16-25) and profound injuries (ISS > 16) had significantly higher sORP measures than the patients classified with mild to moderate injuries (ISS $\leq 15 ; p<0.05$ ). This effect was not found at admission or on day 7 after injury (Figure 3).

Despite the significant correlation, differences in antioxidant capacity did not reach significance between ISS groups ( $p=0.08)$. Patients classified with severe or profound injuries had a trend for higher icORP values than patients with moderate or mild injuries (Figure 3 ). This effect was not 


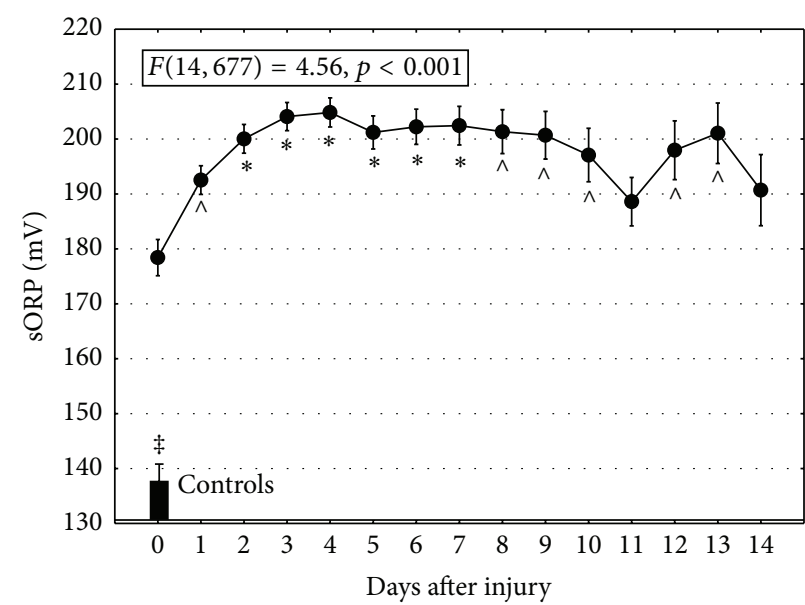

(a)

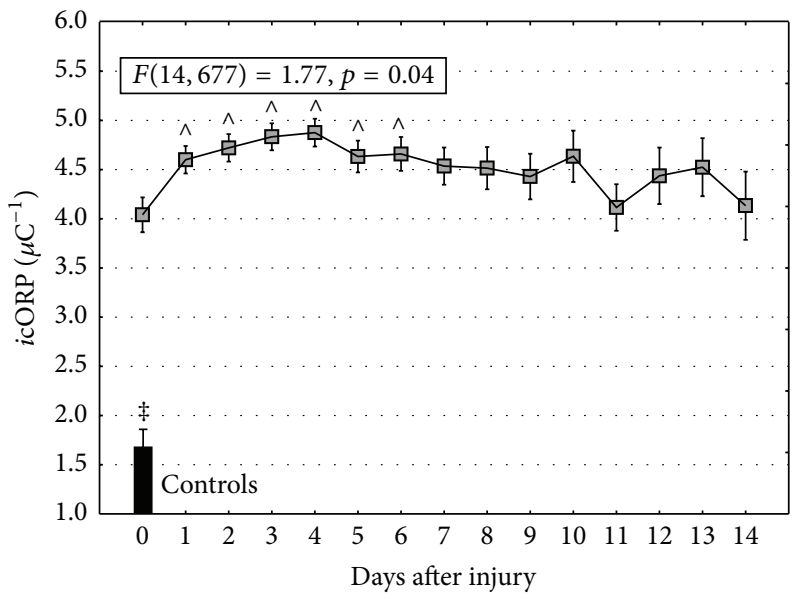

(c)

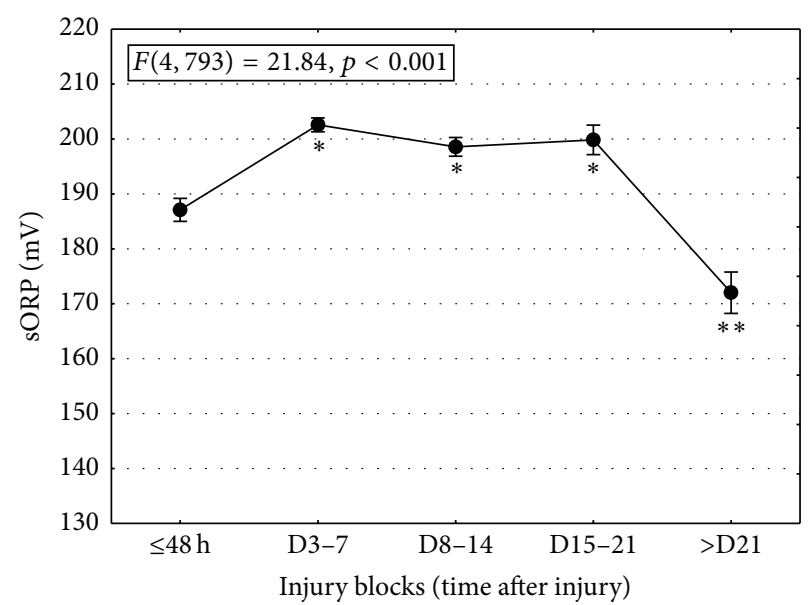

(b)

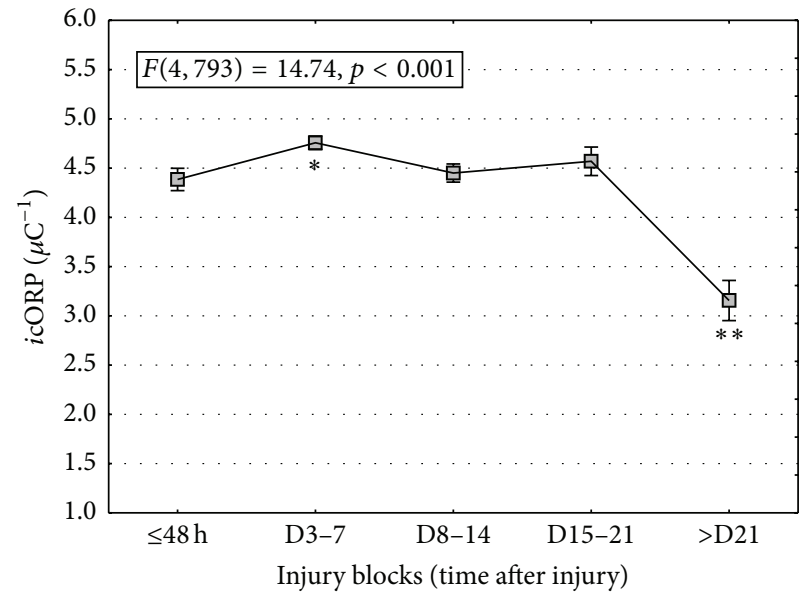

(d)

FIgURE 2: ORP values are higher in TBI than controls and increase in the first few days after injury. (a) Daily changes in sORP indicated significant increases in the first two days after injury and remain reliably high through at least 10 days. Bars represent mean sORP values from healthy controls. (b) Extending the analysis by blocks of time, sORP remains elevated through three weeks (D: days; D15-21). (c) Daily changes in icORP revealed a significant increase (a decrease in antioxidant capacity) in the first day after injury. This steady elevation remains at least through six days after injury. Bars represent mean icORP values from healthy controls. (d) Blocks of data suggested that $i c O R P$ does not decrease (recovery of antioxidant capacity), until three weeks after injury (D15-21). Data are presented as means \pm standard error of the mean (sem); $\wedge$ represents significantly greater than day of injury (day 0$) ; *$ indicates significantly greater than day of injury and day $1 ; * *$ indicates significantly lower than the first 48 hrs after injury; $p<0.05$; means significantly less than TBI.

present in icORP values measured at admission or seven days after injury $(p>0.05)$.

3.5. Type of TBI Injury. Table 2 presents the TBI diagnoses. Approximately $74.3 \%$ of patients had either a subarachnoid, subdural, or epidural hemorrhage. Only two concussion TBI patients had day 7 ORP measures, so this group was not included in day 7 analyses. Analysis of sORP values taken at admission, 4 days after injury, and 7 days after injury did not distinguish between injury types (admission: $F(4,81)=0.84$, $p=0.50 ; 4$ days: $F(4,72)=0.38, p=0.82 ; 7$ days: $F(3,60)=$ $1.14, p=0.34)$. Measures of $i$ CORP also did not distinguish between groups (admission: $F(4,81)=1.79, p=0.14$; day 4 : $F(4,72)=0.94, p=0.45$; day $7: F(3,60)=0.21, p=0.89)$.

\section{Discussion}

The data presented suggests that ORP may be a useful biomarker in TBI. Measures of ORP in TBI patients indicate that after injury they are in a state of oxidative stress, measured by elevated sORP, which is higher in patients with more severe injury. However, and maybe more importantly, is the fact that those patients with an inability to manage the additional oxidative stress, measured by elevated icORP, were more susceptible to a poor acute outcome. Differences in icORP identified, with a high rate of accuracy, the acute outcome of the TBI patients.

Both sORP and icORP increased in the 24-48 hours after injury and remained fairly stable over time regardless of type of TBI, severity, or outcome, except on day 4 where 


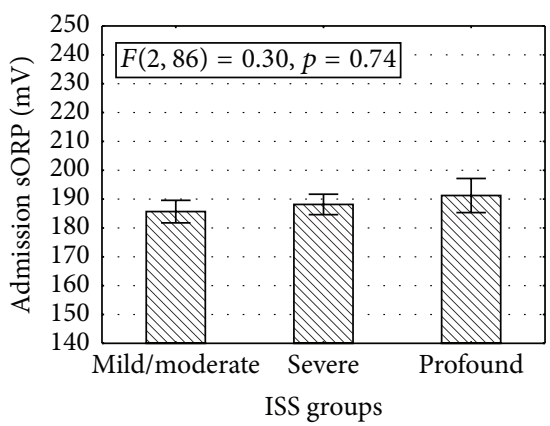

(a)

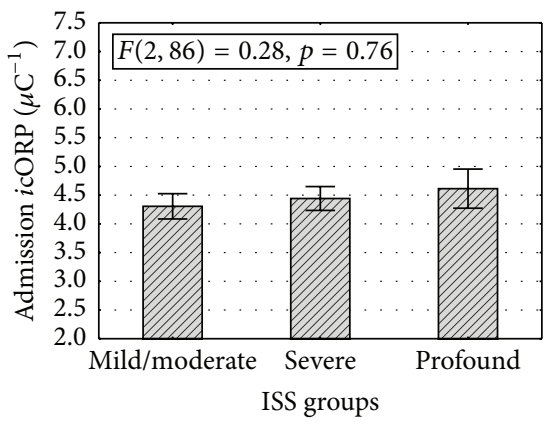

(d)

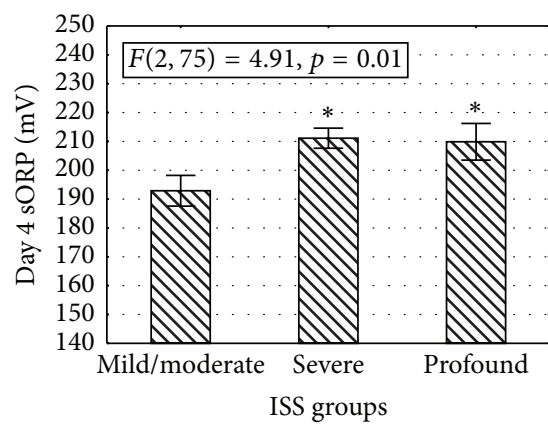

(b)

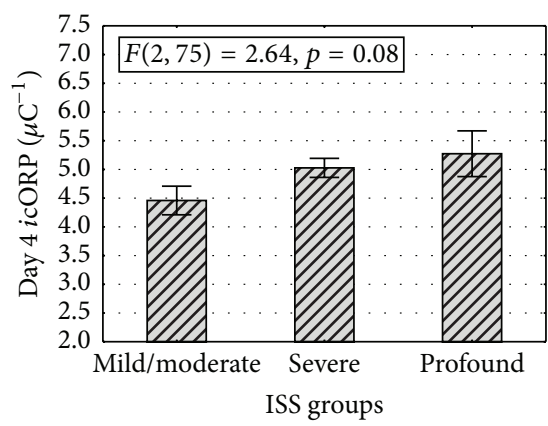

(e)

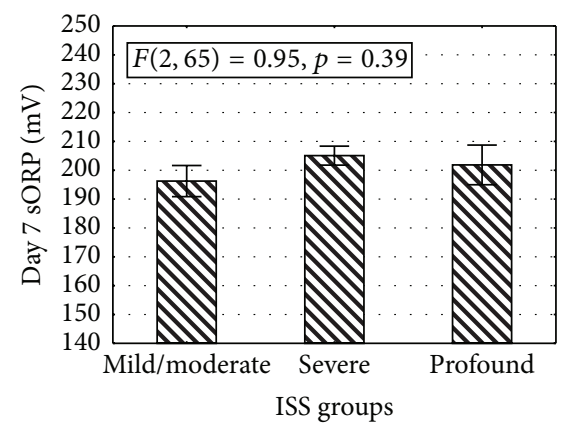

(c)

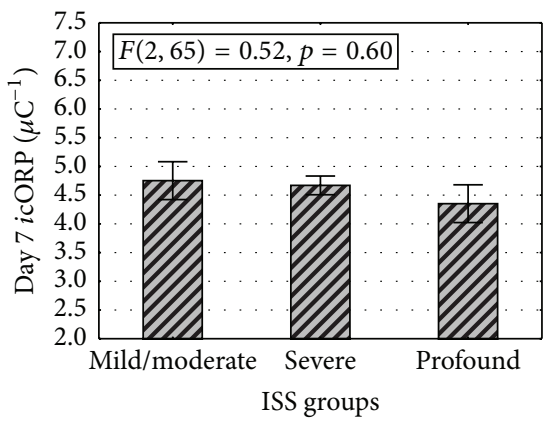

(f)

FIGURE 3: ORP measures increase as a function of ISS groups on the fourth day after injury. (a/d) Admission measures of sORP (a) or icORP (d) did not change as a function of ISS severity. Regardless of severity, groups had similar sORP and icORP values. (b/e) On day 4, sORP measures were significantly higher in the moderate, severe, and profound groups compared to the mild group (b). The severe and profound groups both had increased icORP measures (lowered antioxidant capacity) on day 4 compared to the moderate or mild groups (e). Thus, with increasing level of severity, there were significant increases in sORP and icORP. (c/f) No significant differences were found in ORP measures between moderate, severe, and profound severity groups on day 7 after injury. The mild group did not have enough subjects to be included in this analysis. Data are presented as means \pm standard error of the mean (sem); * means significantly greater than the mild severity group; $p<0.05$.

a significant divergence was measured for acute outcome and severity. Patients with a good acute outcome all had a day 4 icORP value less than or equal to $7.25 \mu \mathrm{C}$. This cut-off value was $96 \%$ accurate in identifying patients by acute outcome. Since the time between hospital admission and death/hospice ranged from 6 to 19 days, measuring icORP on day 4 still would provide valuable information about the probability of a good acute outcome several days to weeks in advance. Predicting outcome by GCS, ISS, or AIS-head score was less successful and pairing icORP values with severity scores did not improve their prognostic power. Relative to other published studies, icORP compares favorably with serumbased Tau, suPAR, and Tau; however measuring ORP has the advantage of providing results more quickly [4, 11, 13, 40-45].

TBI severity, measured by ISS and AIS-head score, was correlated with both measures of ORP on day 4. Day 4 appears to be a crucial time point in the resolution of TBI. A limitation of the study is that the patient sample was drawn from those in which TBI was the primary injury but not necessarily their only injury, that is, isolated TBI. Both the mixed state of the injuries and TBI would contribute to altering the redox balance and elevate ORP values. It is possible then that when the lesser injuries begin to resolve, the contribution to redox imbalance made by TBI alone might be detected, allowing us to distinguish between TBI severities and acute outcomes based on ORP levels on day 4.

We further speculate that because brain injury is a multiphasic response, the ORP measured on day 4 captures these later phases and detects potential differences in the ongoing healing process thus allowing us to identify those with a poor acute outcome. The brain's response to TBI unfolds over the course or hours and days; thus the change in day 4 icORP values may reflect the point at which current antioxidant reserves begin to falter especially in those patients that have a poor acute outcome. Based on controlled animal experiments in TBI, we can establish a timeline of events which may contribute to the depletion of those antioxidant reserves. There is an immediate disruption of the BBB after injury along with the accumulation of neutrophils and the expression of MMP9, myeloperoxidase, and the antiinflammatory cytokine, IL-10 [16, 17, 46-50]. Many of these elevations will decline over the course of several days after injury. Others, like IL6, IL8, MMP2, TNF-p55, and TNF-p75 and the invasion of reactive astrocytes emerge in subsequent days sustaining the state of oxidative stress $[15,16,48,50]$. In addition, the BBB disruption and invading neutrophils have a biphasic response, which could reinitiate the inflammatory reaction and the ensuing oxidative stress [16, 17, 46, 47]. 
The time course for changes in sORP and icORP parallels, especially in the initial days after injury, the emergence of free-radicals, elevated cytokine expression, and neutrophil recruitment.

\section{Conclusion}

The current study supports the notion that ORP can be a valuable tool in assessing injury severity and acute outcome in TBI. A difference in antioxidant capacity, measured by icORP, appears to differentiate between those that will have a good acute outcome from those that will not. sORP, an all-inclusive measure of oxidative stress, was a valuable indicator of injury severity. Interestingly, the projective powers of both sORP and $i$ CORP were found in samples taken four days after injury, emphasizing the importance of the bodily response to injury with time. The types of TBI present in the patient sample had similar levels of ORP.

\section{Competing Interests}

Dr. Bjugstad is a paid employee and Dr. Bar-Or is a paid consultant for Aytu BioScience, Inc. The remaining coauthors declare that they have no competing interests.

\section{Acknowledgments}

The authors gratefully acknowledge the collection of patient samples and maintenance of patient records by Rachel Aumann, RN, and Anita Leyden, RN. This study was supported by Trauma Research, LLC (Englewood, CO), the Swedish Medical Center (Englewood, CO), St. Anthony Central Hospital (Denver, CO), and the Institute for Molecular Medicine (Englewood, CO).

\section{References}

[1] A. Kehoe, S. Rennie, and J. E. Smith, "Glasgow Coma Scale is unreliable for the prediction of severe head injury in elderly trauma patients," Emergency Medicine Journal, vol. 32, no. 8, pp. 613-615, 2015.

[2] K. Salottolo, A. S. Levy, D. S. Slone, C. W. Mains, and D. BarOr, "The effect of age on Glasgow Coma Scale score in patients with traumatic brain injury," JAMA Surgery, vol. 149, no. 7, pp. 727-734, 2014.

[3] J.-I. Ono, A. Yamaura, M. Kubota, Y. Okimura, and K. Isobe, "Outcome prediction in severe head injury: analyses of clinical prognostic factors," Journal of Clinical Neuroscience, vol. 8, no. 2, pp. 120-123, 2001.

[4] F. P. Zemlan, E. C. Jauch, J. J. Mulchahey et al., "C-tau biomarker of neuronal damage in severe brain injured patients: association with elevated intracranial pressure and clinical outcome," Brain Research, vol. 947, no. 1, pp. 131-139, 2002.

[5] G. Teasdale and B. Jennett, "Assessment of coma and impaired consciousness. A practical scale," The Lancet, vol. 304, no. 7872, pp. 81-84, 1974.

[6] M. Faul, L. Xu, M. M. Wald et al., Traumatic Brain Injury in the United States: Emergency Department Visits, Hospitalizations, and Deaths 2002-2006, Centers for Disease Control and
Prevention, National Center for Injury Prevention and Control, 2010.

[7] Report to Congress on Traumatic Brain Injury in the United States: Epidemiology and Rehabilitation, Division of Unintentional Injury Prevention, Centers for Disease Control and Prevention, National Center for Injury Prevention and Control, 2014.

[8] K. Nylén, M. Öst, L. Z. Csajbok et al., "Increased serum-GFAP in patients with severe traumatic brain injury is related to outcome," Journal of the Neurological Sciences, vol. 240, no. 1-2, pp. 85-91, 2006.

[9] C. Yu, A. Boutté, X. Yu et al., "A systems biology strategy to identify molecular mechanisms of action and protein indicators of traumatic brain injury," Journal of Neuroscience Research, vol. 93, no. 2, pp. 199-214, 2015.

[10] L. E. Pelika, A. Kroepfl, M. Leixnering, W. Buchinger, A. Raabe, and H. Redl, "GFAP versus S100B in serum after traumatic brain injury: relationship to brain damage and outcome," Journal of Neurotrauma, vol. 21, no. 11, pp. 1553-1561, 2004.

[11] P.-C. Liliang, C.-L. Liang, H.-C. Weng et al., " $\tau$ proteins in serum predict outcome after severe traumatic brain Injury," Journal of Surgical Research, vol. 160, no. 2, pp. 302-307, 2010.

[12] A. Vilalta, J. Sahuquillo, A. Rosell, M. A. Poca, M. Riveiro, and J. Montaner, "Moderate and severe traumatic brain injury induce early overexpression of systemic and brain gelatinases," Intensive Care Medicine, vol. 34, no. 8, pp. 1384-1392, 2008.

[13] L. Papa, L. Akinyi, M. C. Liu et al., "Ubiquitin C-terminal hydrolase is a novel biomarker in humans for severe traumatic brain injury," Critical Care Medicine, vol. 38, no. 1, pp. 138-144, 2010.

[14] V. A. Tyurin, Y. Y. Tyurina, G. G. Borisenko et al., "Oxidative stress following traumatic brain injury in rats: quantitation of biomarkers and detection of free radical intermediates," Journal of Neurochemistry, vol. 75, no. 5, pp. 2178-2189, 2000.

[15] M. Bains and E. D. Hall, "Antioxidant therapies in traumatic brain and spinal cord injury," Biochimica et Biophysica ActaMolecular Basis of Disease, vol. 1822, no. 5, pp. 675-684, 2012.

[16] A. Chodobski, I. Chung, E. Koźniewska et al., "Early neutrophilic expression of vascular endothelial growth factor after traumatic brain injury," Neuroscience, vol. 122, no. 4, pp. 853867, 2003.

[17] A. Chodobski, B. J. Zink, and J. Szmydynger-Chodobska, "Blood-brain barrier pathophysiology in traumatic brain injury," Translational Stroke Research, vol. 2, no. 4, pp. 492-516, 2011.

[18] K. N. Corps, T. L. Roth, and D. B. McGavern, "Inflammation and neuroprotection in traumatic brain injury," JAMA Neurology, vol. 72, no. 3, pp. 355-362, 2015.

[19] M. Simard and M. Nedergaard, "The neurobiology of glia in the context of water and ion homeostasis," Neuroscience, vol. 129, no. 4, pp. 877-896, 2004.

[20] R. A. Swanson, W. Ying, and T. M. Kauppinen, "Astrocyte influences on ischemic neuronal-death," Current Molecular Medicine, vol. 4, no. 2, pp. 193-205, 2004.

[21] J. M. McCord, "The evolution of free radicals and oxidative stress," American Journal of Medicine, vol. 108, no. 8, pp. 652659, 2000.

[22] S. Vertuani, A. Angusti, and S. Manfredini, "The antioxidants and pro-antioxidants network: an overview," Current Pharmaceutical Design, vol. 10, no. 14, pp. 1677-1694, 2004. 
[23] D. Costantini and S. Verhulst, "Does high antioxidant capacity indicate low oxidative stress?" Functional Ecology, vol. 23, no. 3, pp. 506-509, 2009.

[24] D. Bar-Or, R. Bar-Or, L. T. Rael, and E. N. Brody, "Oxidative stress in severe acute illness," Redox Biology, vol. 4, pp. 340-345, 2015.

[25] S. Milić, J. Bogdanović Pristov, D. Mutavdžić, A. Savić, M. Spasić, and I. Spasojević, "The relationship of physicochemical properties to the antioxidative activity of free amino acids in fenton system," Environmental Science and Technology, vol. 49, no. 7, pp. 4245-4254, 2015.

[26] K. Victorin, K. G. Hellström, and R. Rylander, "Redox potential measurements for determining the disinfecting power of chlorinated water," Journal of Hygiene, vol. 70, no. 2, pp. 313-323, 1972.

[27] K. Victorin, "A field study of some swimming-pool waters with regard to bacteria, available chlorine and redox potential," Journal of Hygiene, vol. 72, no. 1, pp. 101-110, 1974.

[28] World Health Organization, WHO Guidelines for DrinkingWater Quality, WHO Press, 4th edition, 2011.

[29] L. T. Rael, R. Bar-Or, R. M. Aumann, D. S. Slone, C. W. Mains, and D. Bar-Or, "Oxidation-reduction potential and paraoxonase-arylesterase activity in trauma patients," Biochemical and Biophysical Research Communications, vol. 361, no. 2 , pp. 561-565, 2007.

[30] L. T. Rael, R. Bar-Or, C. W. Mains, D. S. Slone, A. S. Levy, and D. Bar-Or, "Plasma oxidation-reduction potential and protein oxidation in traumatic brain injury," Journal of Neurotrauma, vol. 26, no. 8, pp. 1203-1211, 2009.

[31] L. T. Rael, R. Bar-Or, K. Salottolo et al., "Injury severity and serum amyloid A correlate with plasma oxidation-reduction potential in multi-trauma patients: a retrospective analysis," Scandinavian Journal of Trauma, Resuscitation and Emergency Medicine, vol. 17, article 57, 2009.

[32] L. Zhi, X. Hu, and C. Han, "Biphasic changes (overreduction and overoxidation) of plasma redox status and clinical implications in early stage of severe burns," Journal of Critical Care, vol. 29, no. 6, pp. 1063-1068, 2014.

[33] A. Copeland and D. A. Lytle, "Measuring the oxidationreduction potential of important oxidants in drinking water," Journal of the American Water Works Association, vol. 106, no. 1, pp. E10-E20, 2014.

[34] Y. Spanidis, N. Goutzourelas, D. Stagos et al., "Assessment of oxidative stress in septic and obese patients using markers of oxidation-reduction potential," In Vivo, vol. 29, no. 5, pp. 595600, 2015

[35] Y. Spanidis, N. Goutzourelas, D. Stagos et al., "Variations in oxidative stress markers in elite basketball players at the beginning and end of a season," Experimental and Therapeutic Medicine, vol. 11, no. 1, pp. 147-153, 2016.

[36] Y. Spanidis, A. Mpesios, D. Stagos et al., "Assessment of the redox status in patients with metabolic syndrome and type 2 diabetes reveals great variations," Experimental and Therapeutic Medicine, vol. 11, no. 3, pp. 895-903, 2016.

[37] D. Stagos, N. Goutzourelas, D. Bar-Or et al., "Application of a new oxidation-reduction potential assessment method in strenuous exercise-induced oxidative stress," Redox Report, vol. 20, no. 4, pp. 154-162, 2015.

[38] D. Stagos, N. Goutzourelas, A.-M. Ntontou et al., "Assessment of eccentric exercise-induced oxidative stress using oxidationreduction potential markers," Oxidative Medicine and Cellular Longevity, vol. 2015, Article ID 204615, 10 pages, 2015.
[39] L. T. Rael, R. Bar-Or, M. T. Kelly, M. M. Carrick, and D. BarOr, "Assessment of oxidative stress in patients with an isolated traumatic brain injury using disposable electrochemical test strips," Electroanalysis, vol. 27, no. 11, pp. 2567-2573, 2015.

[40] L. Yu, X. Wu, H. Wang, D. Long, J. Yang, and Y. Zhang, "Diagnostic and prognostic significance of suPAR in traumatic brain injury," Neurology India, vol. 62, no. 5, pp. 498-502, 2014.

[41] E. W. Steyerberg, N. Mushkudiani, P. Perel et al., "Predicting outcome after traumatic brain injury: development and international validation of prognostic scores based on admission characteristics," PLoS Medicine, vol. 5, no. 8, pp. 1251-1261, 2008.

[42] P. Kaloostian, C. Robertson, S. P. Gopinath et al., "Outcome prediction within twelve hours after severe traumatic brain injury by quantitative cerebral blood flow," Journal of Neurotrauma, vol. 29, no. 5, pp. 727-734, 2012.

[43] A. D. Walder, P. M. Yeoman, and A. Turnbull, "The abbreviated injury scale as a predictor of outcome of severe head injury," Intensive Care Medicine, vol. 21, no. 7, pp. 606-609, 1995.

[44] P. F. Stahel, "Pupil evaluation in addition to Glasgow Coma Scale components in prediction of traumatic brain injury and mortality," British Journal of Surgery, vol. 99, supplement 1, p. $131,2012$.

[45] M. M. Bembea, N. Rizkalla, J. Freedy et al., "Plasma biomarkers of brain injury as diagnostic tools and outcome predictors after extracorporeal membrane oxygenation," Critical Care Medicine, vol. 43, no. 10, pp. 2202-2211, 2015.

[46] S. A. Baldwin, I. Fugaccia, D. R. Brown, L. V. Brown, and S. W. Scheff, "Blood-brain barrier breach following cortical contusion in the rat," Journal of Neurosurgery, vol. 85, no. 3, pp. 476-481, 1996.

[47] M. K. Başkaya, A. M. Rao, A. Doğan, D. Donaldson, and R. J. Dempsey, "The biphasic opening of the blood-brain barrier in the cortex and hippocampus after traumatic brain injury in rats," Neuroscience Letters, vol. 226, no. 1, pp. 33-36, 1997.

[48] J. S. Truettner, O. F. Alonso, and W. Dalton Dietrich, "Influence of therapeutic hypothermia on matrix metalloproteinase activity after traumatic brain injury in rats," Journal of Cerebral Blood Flow and Metabolism, vol. 25, no. 11, pp. 1505-1516, 2005.

[49] N. C. Royo, F. Wahl, and J.-M. Stutzmann, "Kinetics of polymorphonuclear neutrophil infiltration after a traumatic brain injury in rat," NeuroReport, vol. 10, no. 6, pp. 1363-1367, 1999.

[50] B. Maier, R. Lefering, M. Lehnert et al., "Early versus late onset of multiple organ failure is associated with differing patterns of plasma cytokine biomarker expression and outcome after severe trauma," Shock, vol. 28, no. 6, pp. 668-674, 2007. 


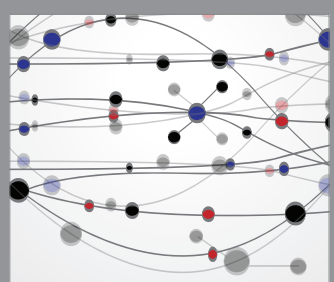

The Scientific World Journal
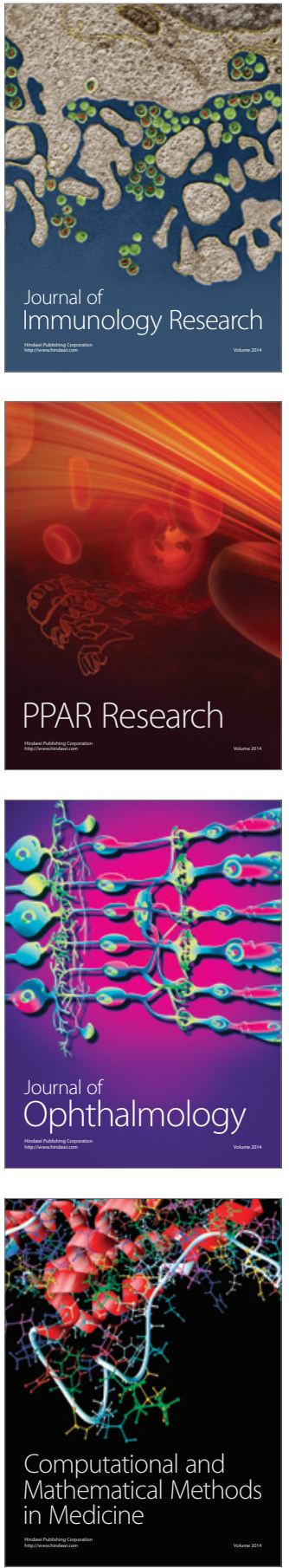

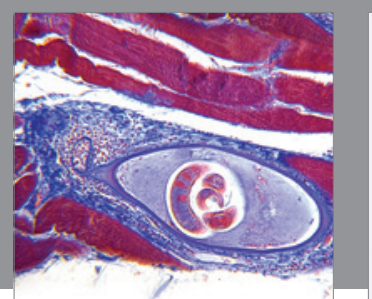

Gastroenterology Research and Practice

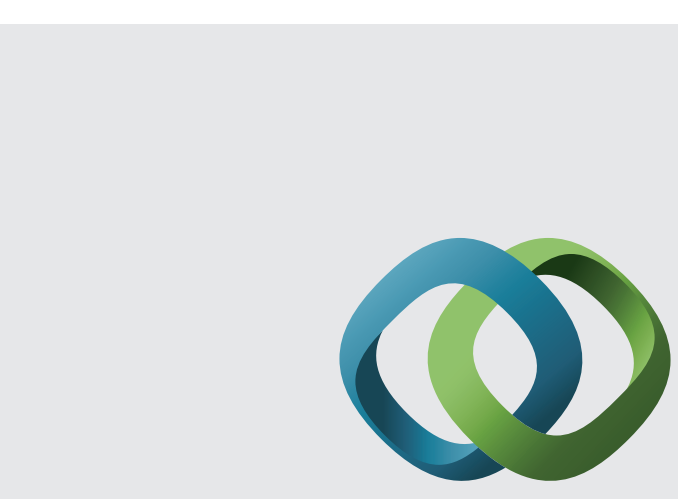

\section{Hindawi}

Submit your manuscripts at

http://www.hindawi.com
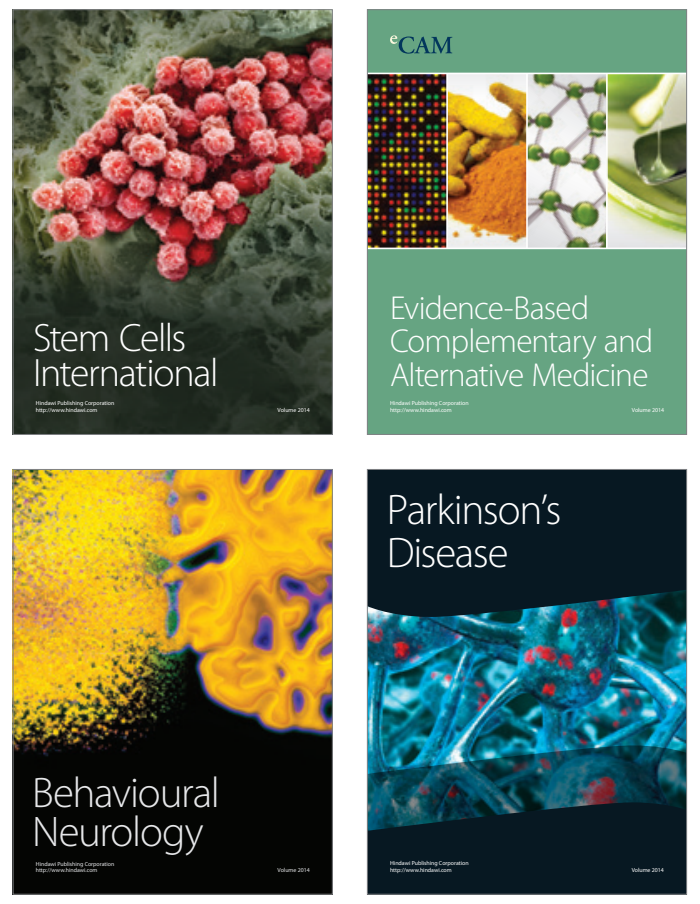
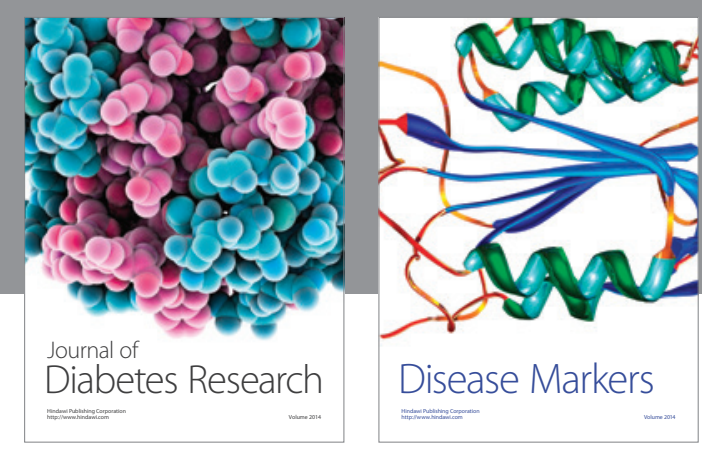

Disease Markers
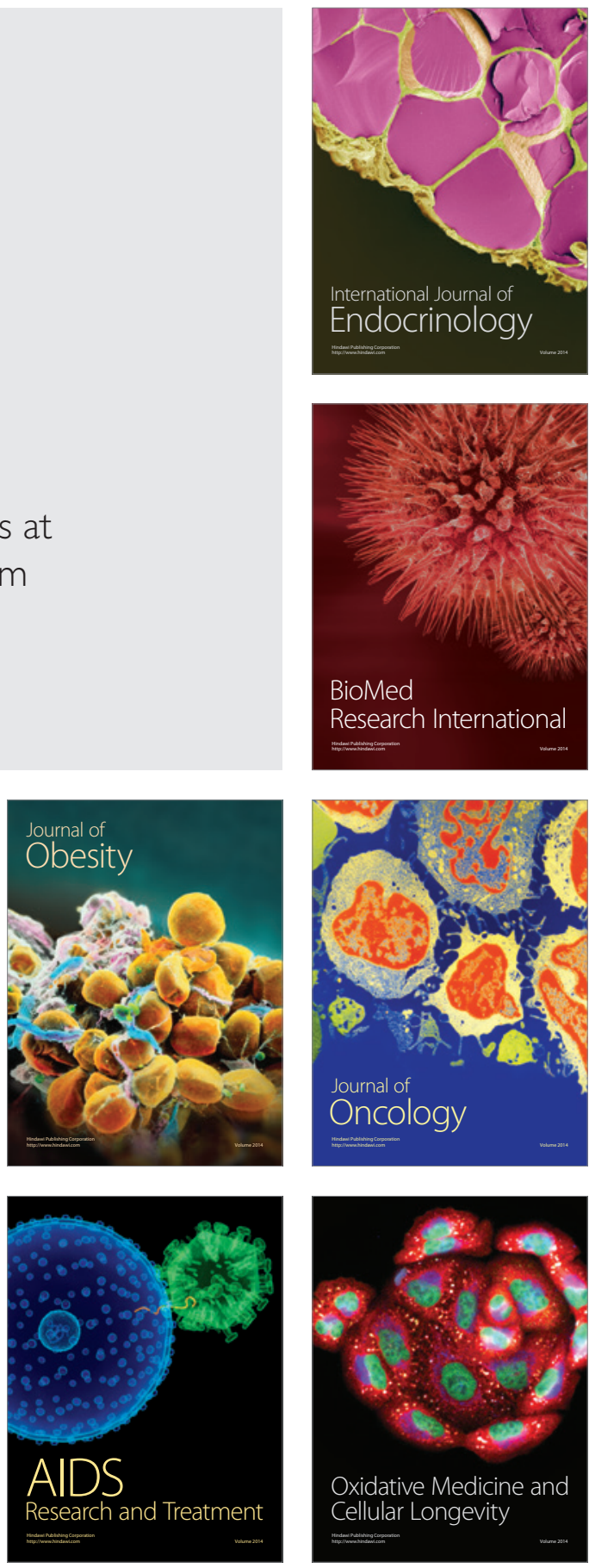